\title{
Theory of Thermal Motion in Electromagnetically Induced Transparency: Diffusion, Doppler, Dicke and Ramsey
}

\author{
O. Firstenberg, ${ }^{1}$ M. Shuker, ${ }^{1}$ R. Pugatch, ${ }^{2}$ D. R. Fredkin, ${ }^{3}$ N. Davidson, ${ }^{2}$ and A. Ron ${ }^{1}$ \\ ${ }^{1}$ Department of Physics, Technion-Israel Institute of Technology, Haifa 32000, Israel \\ ${ }^{2}$ Department of Physics of Complex Systems, Weizmann Institute of Science, Rehovot 76100, Israel \\ ${ }^{3}$ Department of Physics, University of California, San Diego, La Jolla, California 92093
}

We present a theoretical model for electromagnetically induced transparency (EIT) in vapor, that incorporates atomic motion and velocity-changing collisions into the dynamics of the densitymatrix distribution. Within a unified formalism we demonstrate various motional effects, known for EIT in vapor: Doppler-broadening of the absorption spectrum; Dicke-narrowing and time-offlight broadening of the transmission window for a finite-sized probe; Diffusion of atomic coherence during storage of light and diffusion of the light-matter excitation during slow-light propagation; and Ramsey-narrowing of the spectrum for a probe and pump beams of finite-size.

\section{INTRODUCTION}

The Doppler effect, discovered in the mid-19th century, causes a broadening of spectral lines in thermal media which is linearly proportional to the radiation wave-vector [1]. In 1953 R. H. Dicke predicted that the Doppler-broadened spectrum can be narrowed due to frequent velocity-changing collisions [2, 3, 4], by a factor proportional to the ratio between the collisions mean free-path and the radiation wavelength. This phenomenon, known as Dicke narrowing, was observed for microwave and optical transitions 5, 6]. When the motion of the atoms is diffusive, the resulting width is proportional to the diffusion coefficient and quadratic in the radiation wave-vector [4, 7]. Therefore it is sometimes referred to as diffusion-narrowing (of the Doppler profile) or diffusion-broadening (of the spectrum of a stationary atom). For a finite-size beam, as illustrated in Fig. 1, both the Doppler and the Dicke widths can be explained as a time-of-flight (TOF) broadening. A comprehensive literature survey and a theoretical treatment of the Doppler-Dicke problem is presented by May [8].

Electromagnetically Induced Transparency (EIT) occurs when two light fields, a probe and a pump, couple two sublevels of an atomic ground state manifold to a common upper level. When the Raman resonance conditions are satisfied, a quantum coherence evolves within the ground state in the process of coherent population trapping (CPT) [9], inducing a unique transparency window in the absorption spectrum, accompanied by dispersion. The spectral width of these features depends primarily on the ground-state decoherence rates, ranging from the order of several $\mathrm{Hz}$ in cryogenically-cooled crystals [10] up to the order of several $\mathrm{KHz}$ or $\mathrm{MHz}$ in thermal vapor [11]. A wide variety of phenomena has been demonstrated utilizing the ultra-narrow EIT resonances, e.g. slow light [12], stored light [13], and non-linear optics at low light levels 14. Having the advantage of being relatively simple to implement, thermal vapor EIT has been used for various applications, such as frequency standards [15, 16], magnetometers [17] and optical delay lines. In this work we present a comprehensive ana-

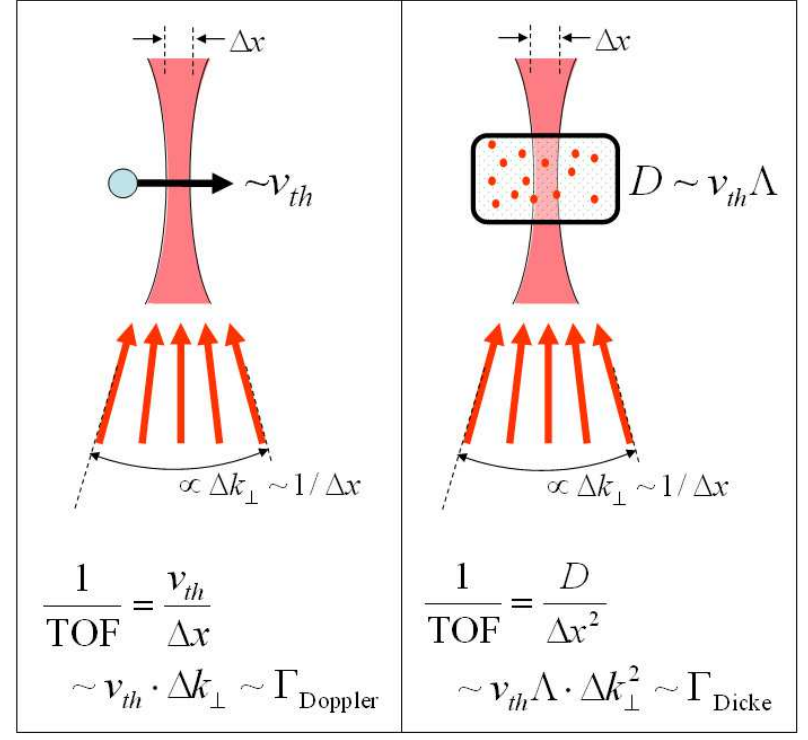

FIG. 1: Illustration of time-of-flight (TOF) broadening in the Doppler (left) and Dicke (right) limits. Assume a beam of width $\Delta x$ in the transverse plane and width $\Delta k_{\perp} \sim 1 / \Delta x$ in $k$-space. Atoms with a transverse velocity $v_{t h}$ (left) cross the beam in time $\Delta x / v_{t h}$ and cause a TOF broadening of the order of $v_{t h} \Delta k_{\perp}$, which is equal to the well-known Dopplerwidth. Atoms that undergo diffusion (right), traverse the beam in average time of $\Delta x^{2} / D$, where $D=v_{t h} \Lambda$ is the diffusion coefficient and $\Lambda$ is the mean-free path between collisions. This results in a TOF broadening of the order of $v_{t h} \Lambda \Delta k_{\perp}^{2}$, which is the well-known Dicke-width.

lytic model for the effect of atomic motion on vapor EIT, describing broadening and narrowing mechanisms, and the phenomena of diffusion during slowing and storage of light, within a unified formalism.

As a two-photon process, EIT in room-temperature vapor is susceptible to residual Doppler broadening, proportional to $\delta q=\left|\mathbf{q}_{1}-\mathbf{q}_{2}\right|$, where $\mathbf{q}_{1}$ and $\mathbf{q}_{2}$ are the wavevectors of the probe and the pump, respectively. However in the presence of buffer gas, when diffusion dominates the atomic motion, a Dicke-like narrowing of the Doppler 
spectrum may occur and the narrowing factor is proportional to the ratio between the mean-free path and $\delta q^{-1}$ [18]. In an hyperfine EIT, $\delta q^{-1}$ is of the order of centimeters and the narrowing factor is of the order $10^{-4}$, completely diminishing the residual Doppler effect and allowing the high accuracy of EIT-based frequency standards [15, 19]. By introducing a small angular deviation between the probe and the pump, it is possible to quantitatively measure the residual-Doppler width and the Dicke width of Zeeman EIT lines [20, 21].

For probe and pump beams of finite size, the EIT spectrum is subjected also to TOF broadening [22]. Here again, the broadening reduces to the Dicke-type in the limit of diffusional motion. Nevertheless, the spectrum is also affected by a more complicated mechanism, recently denoted as Ramsey narrowing, which is attributed to the random-walk of atoms in and out of the beams [23, 24]. The process in which an atom carrying groundstate coherence leaves the beam, evolves "in the dark", and then re-enter the beam, is analogous to the Ramsey method of separated oscillating fields [25]. This process is more relevant for two-photon phenomena, such as velocity-selective optical pumping, magneto-optical spectroscopy and CPT/EIT, because of the small frequencies $(c \cdot \delta q)$ and long coherence times, that are comparable with the typical time the atoms spend inside and outside the beam. Ramsey-like features in such systems were observed in the velocity, time and space domains [26, 27, 28, 29]. Ramsey narrowing occurs due to the random distribution of the durations "in the dark", resulting in a superposition of the spectral Ramsey fringes, that wipes out the fringes and leaves a single narrow feature in the center. A theoretical technique of integrating over all possible Ramsey sequences, taking into account calculated distributions of the durations in and out of the beams, agrees well with experimental results [24].

While Ramsey narrowing is an evidence for the diffusion of EIT coherence, a more explicit demonstration was given in recent storage-of-light experiments, in which an elaborated spatial profile of the probe field was stored and retrieved in vapor [30, 31]. The restored profiles were exactly predicted by assuming that during storage, when no fields are present, the ground-state coherence undergoes regular diffusion. Since the coherence is complex, the phase pattern also diffused and interference effects were observed. Neither a more basic theory that derives the diffusion equation for the atomic coherence in the absence of fields nor a prediction for the diffusion-like behavior of slow-light were presented.

The model presented in this paper describes the effect of velocity-changing collisions on the position and velocity dependence of the atomic density matrix. Assuming a Boltzmann-like relaxation in velocity space, we write in section Пthe dynamics equations for the internal and the external atomic motion, under the weak-probe approximation. We then derive the equations of motion for the slowly-varying envelopes of the atomic coherences and the probe's field, while the pump's envelope is as- sumed stationary. In section III we formally solve these equations and derive the probe's susceptibility, for the general case and for a plane-wave pump. By general we mean that neither the "Doppler" nor the "Dicke" limits are taken [8] for the one-photon or the two-photon transitions. This model extends our previous study on Dicke-narrowing [18] in the following aspects: it avoids the use of the low-contrast approximation, incorporating the power-broadening effect; it allows for a non-planar (finite-size) probe beam, introducing a wave-vector filter; it is time-dependent and thus allows for the propagation of a probe package. We note also that the assumption of Gaussian distribution of atomic trajectories is avoided here (see Eq. (9) in Ref. [18]). In section [V we consider the realistic regime, where the velocity relaxation-rate is large enough to cause Dicke-narrowing of the two-photon transition. In this regime we derive a diffusion equation for the density-matrix distribution during storage-of-light and afterwards calculate the dynamics in the presence of the fields. For the case of plane-wave pump we recover the Dicke-Diffusion absorption spectrum and arrive at a diffusion-diffraction equation for the slowly propagating envelope of the probe. For the case of finite-size pump and probe, analyzed in section $\nabla$, we solve the diffusion equations and retrieve the Ramsey-narrowed absorption spectrum. Note that the latter is done in a steadystate approach, rather then by averaging over all possible atomic trajectories.

\section{EQUATIONS OF MOTION}

\section{A. Atom-Field Interaction}

We consider three-level atoms in a $\Lambda$-configuration, with an upper state $|3\rangle$ and two lower states, $|2\rangle$ and $|1\rangle$, as depicted in Fig. 2. The atoms interact with two external, classical electromagnetic fields, propagating in time $t$ and space $\mathbf{r}$,

$$
\mathbf{E}_{s}(\mathbf{r}, t)=\Re e\left\{\frac{\hbar}{\mu_{3 s}} \epsilon_{s} \Omega_{s}(\mathbf{r}, t)\right\} \text { for } s=1,2,
$$

with

$$
\Omega_{s}(\mathbf{r}, t)=\tilde{\Omega}_{s}(\mathbf{r}, t) e^{-i \omega_{s} t} e^{i \mathbf{q}_{s} \cdot \mathbf{r}},
$$

where $\epsilon_{s}$ and $\mathbf{q}_{s}$ are the polarization vector and wave vector of the probe $(s=1)$ and the pump $(s=2)$; $\omega_{s}=c\left|\mathbf{q}_{s}\right| ; \mu_{3 s}=\left\langle 3\left|\mathbf{d} \cdot \epsilon_{s}\right| s\right\rangle$ is the $3 \rightarrow s$ transition dipole moment matrix element; and $\widetilde{\Omega}_{s}(\mathbf{r}, t)$ is the slowly varying envelope in time of the Rabi frequency, satisfying $\left|\frac{\partial}{\partial t} \Omega_{s}(\mathbf{r}, t)\right| \ll \omega_{s}\left|\tilde{\Omega}_{s}(\mathbf{r}, t)\right|$.

First we consider a single atomic system, the $i$-th atom, one of many identical particles. The center of mass position $\mathbf{r}_{i}(t)$ enters the internal dynamics of the atom because the external fields must be evaluated there. We denote the $s s^{\prime}$ reduced density-matrix element of the $i$-th atom as $\rho_{s s^{\prime}}^{i}(t)$. For a weak probe, namely when 
(a)

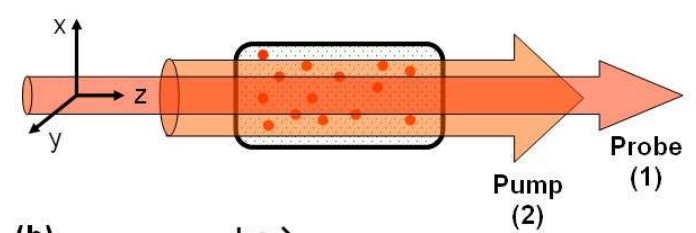

(b)

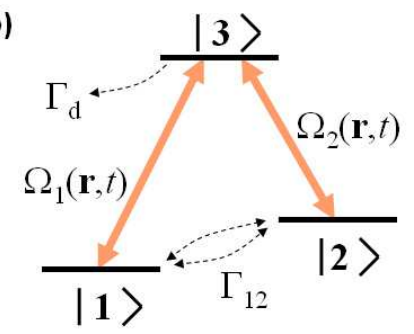

FIG. 2: (a) A probe beam and a pump beam, with a finite envelope in space, propagate through the vapor cell. The $z$-axis is chosen perpendicular to the probe's direction and the $x$ and $y$ axes form the transverse plane. (b) Atomic levels diagram. $\Omega_{1}$ and $\Omega_{2}$ are the Rabi frequencies of the probe and the pump, respectively. $\Gamma_{d}$ and $\Gamma_{12}$ are the decoherence rates of the optical and the ground-state transitions.

the probe field is weak enough to cause $\rho_{31}^{i} \ll \rho_{11}^{i}$, the equations of motion of $\rho_{31}^{i}$ and $\rho_{21}^{i}$ are (cf. [18])

$$
\begin{aligned}
\frac{\partial}{\partial t} \rho_{31}^{i} & =-\Gamma_{d} \rho_{31}^{i}-i \omega_{31} \rho_{31}^{i}+i \Omega_{2}\left(\mathbf{r}_{i}, t\right) \rho_{21}^{i} \\
& +i \Omega_{1}\left(\mathbf{r}_{i}, t\right) \rho_{11}^{i,(\mathrm{eq})} \\
\frac{\partial}{\partial t} \rho_{21}^{i} & =-\Gamma_{21} \rho_{21}^{i}-i \omega_{21} \rho_{21}^{i}+i \Omega_{2}^{*}\left(\mathbf{r}_{i}, t\right) \rho_{31}^{i}
\end{aligned}
$$

Here $\omega_{31}$ and $\Gamma_{d}$ are the frequency and the decoherence rate of the $3 \rightarrow 1$ transition; $\omega_{21}$ and $\Gamma_{21}$ are the frequency and the decoherence rate of the $2 \rightarrow 1$ transition; $\rho_{31}^{i}$ is linear in the probe field; and the equilibrium density matrix in the absence of the probe $\left(\Omega_{1}=0\right)$ is

$$
\rho_{s s^{\prime}}^{i,(\mathrm{eq})}= \begin{cases}1 & s=s^{\prime}=1 \\ 0 & \text { otherwise. }\end{cases}
$$

We consider the case of non-depleted pump, i.e. we assume that the pump's envelope is constant in time and is given by $\tilde{\Omega}_{2}(\mathbf{r}, t)=\tilde{\Omega}_{2}(\mathbf{r})$. The wave equation of the probe field is

$$
\left(\nabla^{2}-\frac{1}{c^{2}} \frac{\partial^{2}}{\partial t^{2}}\right) \mathbf{E}_{1}(\mathbf{r}, t)=\frac{4 \pi}{c^{2}} \frac{\partial^{2}}{\partial t^{2}} \mathbf{P}_{31}(\mathbf{r}, t)
$$

where $\mathbf{P}_{31}(\mathbf{r}, t)=\Re e\left\{\tilde{\mathbf{P}}_{31}(\mathbf{r}, t) e^{-i \omega_{1} t} e^{i \mathbf{q}_{1} \cdot \mathbf{r}}\right\}$ is the contribution of the $3 \rightarrow 1$ transition to the expectation value of the polarization density, and $\tilde{\mathbf{P}}_{31}(\mathbf{r}, t)$ is the slowly varying polarization. With Eq.(1) we have

$$
\left(\frac{\partial}{\partial t}+c \frac{\partial}{\partial z}-i \frac{c}{2 q_{1}} \nabla^{2}\right) \tilde{\Omega}_{1}(\mathbf{r}, t)=i \frac{g}{\mu_{31}^{*}} \epsilon_{1} \cdot \tilde{\mathbf{P}}_{31}(\mathbf{r}, t),
$$

where $g=2 \pi \omega_{1}\left|\mu_{31}\right|^{2} / \hbar$ is a coupling constant and $\nabla^{2}$ is the three-dimensional Laplacian operator. To obtain Eq.(6) we neglected the second order temporal derivatives of the envelopes and chose, without loss of generality, $\mathbf{q}_{1}=\hat{\mathbf{z}} q_{1}$, where $\hat{\mathbf{z}}$ is a unit vector in the $z$-direction (see Fig. 2]a). The second order spatial derivatives are retained to allow the description of finite-size beams.

\section{B. Boltzmann-like Equations}

We consider a dilute gas of active atoms in the presence of a noble buffer-gas that causes frequent velocitychanging coherence-preserving collisions. We introduce a generalized density-matrix distribution function in space and velocity

$\rho_{s s^{\prime}}=\rho_{s s^{\prime}}(\mathbf{r}, \mathbf{v}, t)=\sum_{i} \rho_{s s^{\prime}}^{i}(t) \delta\left(\mathbf{r}-\mathbf{r}_{i}(t)\right) \delta\left(\mathbf{v}-\mathbf{v}_{i}(t)\right)$,

where the time dependence of $\rho_{s s^{\prime}}^{i}(t)$ is due to the internal dynamics only, considered earlier in the singleparticle density-matrix. We express

$$
\begin{aligned}
\frac{\partial}{\partial t} \rho_{s s^{\prime}} & =\sum_{i}\left(\frac{\partial}{\partial t} \rho_{s s^{\prime}}^{i}\right) \delta\left(\mathbf{r}-\mathbf{r}_{i}(t)\right) \delta\left(\mathbf{v}-\mathbf{v}_{i}(t)\right) \\
& +\sum_{i} \rho_{s s^{\prime}}^{i} \frac{d \mathbf{r}_{i}}{d t} \cdot\left[\frac{\partial}{\partial \mathbf{r}_{i}} \delta\left(\mathbf{r}-\mathbf{r}_{i}(t)\right)\right] \delta\left(\mathbf{v}-\mathbf{v}_{i}(t)\right) \\
& +\sum_{i} \rho_{s s^{\prime}}^{i} \delta\left(\mathbf{r}-\mathbf{r}_{i}(t)\right) \frac{d \mathbf{v}_{i}}{d t} \cdot\left[\frac{\partial}{\partial \mathbf{v}_{i}} \delta\left(\mathbf{v}-\mathbf{v}_{i}(t)\right)\right]
\end{aligned}
$$

or

$$
\begin{aligned}
& \frac{\partial}{\partial t} \rho_{s s^{\prime}}+\mathbf{v} \cdot \frac{\partial}{\partial \mathbf{r}} \rho_{s s^{\prime}}+\left[\frac{\partial}{\partial t} \rho_{s s^{\prime}}\right]_{\mathrm{col}} \\
& =\sum_{i}\left(\frac{\partial}{\partial t} \rho_{s s^{\prime}}^{i}\right) \delta\left(\mathbf{r}-\mathbf{r}_{i}(t)\right) \delta\left(\mathbf{v}-\mathbf{v}_{i}(t)\right)
\end{aligned}
$$

where $\left[\frac{\partial}{\partial t} \rho_{s s^{\prime}}\right]_{\text {col }}$ is the effect of collisions. Notice that the density-matrix distribution function is classical, as far as its external motion is concerned, and quantum mechanical in its internal atomic motion. The function $\rho_{s s^{\prime}}(\mathbf{r}, \mathbf{v}, t) d^{3} r d^{3} v$ is interpreted as the probability to find an atom of density-matrix element $\rho_{s s^{\prime}}$ at the time $t$, near position $\mathbf{r}$, within a volume element $d^{3} r$, with velocity $\mathbf{v}$, within a velocity volume element $d^{3} v$.

With this interpretation we understand the effect of collisions as relaxation to thermal equilibrium of the center of mass, and we shall take it into account using the Boltzmann relaxation method. Introducing the density

$$
R_{s s^{\prime}}(\mathbf{r}, t)=\int d^{3} v \rho_{s s^{\prime}}(\mathbf{r}, \mathbf{v}, t),
$$

which is interpreted as the number of atoms with $\rho_{s s^{\prime}}$ per unit volume near $\mathbf{r}$ in space, we assume that the 
distribution $\rho_{s s^{\prime}}(\mathbf{r}, \mathbf{v}, t)$ relaxes to equilibrium as [4]

$$
\left[\frac{\partial}{\partial t} \rho_{s s^{\prime}}(\mathbf{r}, \mathbf{v}, t)\right]_{\mathrm{col} .}=-\gamma\left[\rho_{s s^{\prime}}(\mathbf{r}, \mathbf{v}, t)-R_{s s^{\prime}}(\mathbf{r}, t) F(\mathbf{v})\right]
$$

Here $\gamma$ is the relaxation rate in time, proportional to the collisions rate, and $F(\mathbf{v})$ is the thermal equilibrium Boltzmann distribution function in velocity space,

$$
F(\mathbf{v})=\frac{1}{\left(2 \pi v_{\mathrm{th}}^{2}\right)^{3 / 2}} e^{-v^{2} /\left(2 v_{\mathrm{th}}^{2}\right)} \quad ; \quad v_{\mathrm{th}}^{2}=\frac{k_{B} T}{m},
$$

where $k_{B}$ is the Boltzmann constant, $T$ is the temperature, $m$ is the atomic mass and $v_{\text {th }}$ is the thermal velocity. Here the internal motion, and the external one, are completely separated. The collisions with the buffer gas affect only the external degrees of freedom and are assumed much frequent than the collisions with the active atoms. If the buffer gas particles affect the internal motion, e.g. by pressure-broadening [7], it is taken into account in the atomic decay rates. The collision term, Eq.(10), neglects partial wave scattering for $l>0$ and also neglects the energy dependence of the scattering cross-section. From Eqs. (3.48) and the Boltzmann collision term of Eq.(10), we observe that

$$
\sum_{i} \rho_{11}^{i,(\mathrm{eq})} \delta\left(\mathbf{r}-\mathbf{r}_{i}(t)\right) \delta\left(\mathbf{v}-\mathbf{v}_{i}(t)\right)=n_{0} F(\mathbf{v}),
$$

where $n_{0}$ is the gas particle density, and the equations of motion are:

$$
\begin{aligned}
&\left(\frac{\partial}{\partial t}+\Gamma_{d}+i \omega_{31}\right.\left.+\mathbf{v} \cdot \frac{\partial}{\partial \mathbf{r}}\right) \rho_{31}-i \Omega_{2}(\mathbf{r}) \rho_{21} \\
&-i \Omega_{1}(\mathbf{r}, t) n_{0} F(\mathbf{v})+\gamma\left[\rho_{31}-R_{31}(\mathbf{r}, t) F(\mathbf{v})\right]=0 \\
&\left(\frac{\partial}{\partial t}+\Gamma_{21}+i \omega_{21}+\mathbf{v} \cdot \frac{\partial}{\partial \mathbf{r}}\right) \rho_{21}-i \Omega_{2}^{*}(\mathbf{r}) \rho_{31} \\
&+\gamma\left[\rho_{21}-R_{21}(\mathbf{r}, t) F(\mathbf{v})\right]=0
\end{aligned}
$$

with $\rho_{21}=\rho_{21}(\mathbf{r}, \mathbf{v}, t)$ and $\rho_{31}=\rho_{31}(\mathbf{r}, \mathbf{v}, t)$. We note that the above semiclassical analysis of the dynamics has a quantum-mechanical equivalent, which will be of significance in a regime where the atomic recoil velocity is comparable with the thermal velocity.

\section{Envelope Equations}

Since the probe field propagates through the cell with a slowly varying envelope, $\rho_{31}(\mathbf{r}, \mathbf{v}, t)$ and $\rho_{21}(\mathbf{r}, \mathbf{v}, t)$ can be expressed as

$$
\begin{aligned}
& \rho_{31}(\mathbf{r}, \mathbf{v}, t)=\tilde{\rho}_{31}(\mathbf{r}, \mathbf{v}, t) e^{-i \omega_{1} t} e^{i \mathbf{q}_{1} \cdot \mathbf{r}}, \\
& \rho_{21}(\mathbf{r}, \mathbf{v}, t)=\tilde{\rho}_{21}(\mathbf{r}, \mathbf{v}, t) e^{-i\left(\omega_{1}-\omega_{2}\right) t} e^{i\left(\mathbf{q}_{1}-\mathbf{q}_{2}\right) \cdot \mathbf{r}},
\end{aligned}
$$

where $\tilde{\rho}_{31}$ and $\tilde{\rho}_{21}$ are slowly varying in space and time. Similarly we introduce the slowly varying densities, $\tilde{R}_{21}(\mathbf{r}, t)$ and $\tilde{R}_{31}(\mathbf{r}, t)$, and express the expectation value of the polarization density, $\tilde{\mathbf{P}}_{31}(\mathbf{r}, t)$, in terms of the density $\tilde{R}_{31}(\mathbf{r}, t)$ as

$$
\epsilon_{1} \cdot \tilde{\mathbf{P}}_{31}(\mathbf{r}, t)=\mu_{31}^{*} \tilde{R}_{31}(\mathbf{r}, t) .
$$

With the one-photon detuning, $\Delta_{1}=\omega_{1}-\omega_{31}$, and the two-photon Raman detuning, $\Delta=\omega_{1}-\omega_{2}-\omega_{21}$, we define

$$
\begin{aligned}
& \xi_{1}=\Delta_{1}-\mathbf{q}_{1} \cdot \mathbf{v}+i\left(\Gamma_{d}+\gamma\right), \\
& \xi_{2}=\Delta-\left(\mathbf{q}_{1}-\mathbf{q}_{2}\right) \cdot \mathbf{v}+i\left(\Gamma_{21}+\gamma\right) .
\end{aligned}
$$

and write Eqs.(13) and Eq.(6) as

$$
\begin{array}{r}
\left(\frac{\partial}{\partial t}+\mathbf{v} \cdot \frac{\partial}{\partial \mathbf{r}}-i \xi_{1}\right) \tilde{\rho}_{31}(\mathbf{r}, \mathbf{v}, t)-\gamma \tilde{R}_{31}(\mathbf{r}, t) F(\mathbf{v}) \\
-i \tilde{\Omega}_{2}(\mathbf{r}) \tilde{\rho}_{21}(\mathbf{r}, \mathbf{v}, t)-i \tilde{\Omega}_{1}(\mathbf{r}, t) n_{0} F(\mathbf{v})=0 \\
\left(\frac{\partial}{\partial t}+\mathbf{v} \cdot \frac{\partial}{\partial \mathbf{r}}-i \xi_{2}\right) \tilde{\rho}_{21}(\mathbf{r}, \mathbf{v}, t)-\gamma \tilde{R}_{21}(\mathbf{r}, t) F(\mathbf{v}) \quad(17 \mathrm{~b}) \\
-i \tilde{\Omega}_{2}^{*}(\mathbf{r}) \tilde{\rho}_{31}(\mathbf{r}, \mathbf{v}, t)=0
\end{array}
$$

and

$$
\left(\frac{\partial}{\partial t}+c \frac{\partial}{\partial z}-i \frac{c}{2 q_{1}} \nabla^{2}\right) \tilde{\Omega}_{1}(\mathbf{r}, t)=i g \tilde{R}_{31}(\mathbf{r}, t) .
$$

Eqs.(17) and (18) compose the full set of equations of motion for the slowly varying envelopes.

Finally, in sections III and IV we study the case of a stationary, plane-wave pump, $\tilde{\Omega}_{2}(\mathbf{r})=\Omega_{2}$. For this case it is convenient introduce the Fourier transformation and replace the slowly varying time dependent and $\mathbf{r}$-dependent functions by

$$
f(\mathbf{r}, t)=\int_{-\infty}^{\infty} \frac{d^{3} k}{2 \pi} e^{i \mathbf{k r}} \int_{-\infty}^{\infty} \frac{d \omega}{2 \pi} e^{-i \omega t} f(\mathbf{k}, \omega)
$$

and write Eqs.(17) as

$$
\begin{array}{r}
\left(\omega-\mathbf{k} \cdot \mathbf{v}+\xi_{1}\right) \tilde{\rho}_{31}(\mathbf{k}, \mathbf{v}, \omega)-i \gamma \tilde{R}_{31}(\mathbf{k}, \omega) F(\mathbf{v}) \quad(20 \mathrm{a}) \\
+\Omega_{2} \tilde{\rho}_{21}(\mathbf{k}, \mathbf{v}, \omega)+\tilde{\Omega}_{1}(\mathbf{k}, \omega) n_{0} F(\mathbf{v})=0 \\
\left(\omega-\mathbf{k} \cdot \mathbf{v}+\xi_{2}\right) \tilde{\rho}_{21}(\mathbf{k}, \mathbf{v}, \omega)-i \gamma \tilde{R}_{21}(\mathbf{k}, \omega) F(\mathbf{v}) \quad(20 \mathrm{~b}) \\
+\Omega_{2}^{*} \tilde{\rho}_{31}(\mathbf{k}, \mathbf{v}, \omega)=0,
\end{array}
$$

and Eq. (18) as

$$
\left(i k_{z}-i \frac{\omega}{c}+i \frac{k^{2}}{2 q_{1}}\right) \tilde{\Omega}_{1}(\mathbf{k}, \omega)=i \frac{g}{c} \tilde{R}_{31}(\mathbf{k}, \omega) .
$$

\section{GENERAL SOLUTION}

We consider the EIT medium in a cell, with a probe beam of finite width in the transverse plane $(x, y)$, propagating along the $z$-axis in the direction of $\mathbf{q}_{1}=\hat{\mathbf{z}} q_{1}$. The variation of $\tilde{\Omega}_{1}$ in space is much slower than $2 \pi / q_{1}$ 
and the variation in time is much slower than $2 \pi / \omega_{1}$. The pump is a plane-wave, $\Omega_{2}$, propagating with a wavevector $\mathbf{q}_{2}$. Here we present the general solution for the probe field propagation inside the cell, i.e. away from its boundaries, for any relaxation rate $\gamma$, without taking the "Doppler" or the "Dicke" limits.

We start from Eqs.(20) and formally solve for $\tilde{\rho}_{31}$ and $\tilde{\rho}_{21}$ :

$$
\tilde{\rho}_{31}=\frac{F(\mathbf{v})}{\xi_{d}}\left|\begin{array}{cc}
i \gamma \tilde{R}_{31}-\tilde{\Omega}_{1}(\mathbf{k}, \omega) n_{0} & \Omega_{2} \\
i \gamma \tilde{R}_{21} & \omega-\mathbf{k v}+\xi_{2}
\end{array}\right|,
$$

and

$$
\tilde{\rho}_{21}=\frac{F(\mathbf{v})}{\xi_{d}}\left|\begin{array}{cc}
\omega-\mathbf{k} \cdot \mathbf{v}+\xi_{1} & i \gamma \tilde{R}_{31}-\tilde{\Omega}_{1}(\mathbf{k}, \omega) n_{0} \\
\Omega_{2}^{*} & i \gamma \tilde{R}_{21}
\end{array}\right|,
$$

where $|\cdots|$ stands for matrix-determinant and

$$
\xi_{d}=\left(\omega-\mathbf{k} \cdot \mathbf{v}+\xi_{1}\right)\left(\omega-\mathbf{k} \cdot \mathbf{v}+\xi_{2}\right)-\left|\Omega_{2}\right|^{2} .
$$

Integrating over velocity, we get coupled equations for $\tilde{R}_{31}$ and $\tilde{R}_{21}$,

$$
\left[\begin{array}{cc}
1-i \gamma G_{1} & i \gamma \tilde{\Omega}_{2} G \\
i \gamma \tilde{\Omega}_{2}^{*} G & 1-i \gamma G_{2}
\end{array}\right]\left[\begin{array}{c}
\tilde{R}_{31} \\
\tilde{R}_{21}
\end{array}\right]=\left[\begin{array}{c}
-G_{1} \\
\Omega_{2}^{*} G
\end{array}\right] \tilde{\Omega}_{1}(\mathbf{k}, \omega) n_{0}
$$

where we have introduced the integrals

$$
\begin{aligned}
G(\mathbf{k}, \omega) & =\int d^{3} v F(\mathbf{v}) \frac{1}{\xi_{d}}, \\
G_{1}(\mathbf{k}, \omega) & =\int d^{3} v F(\mathbf{v}) \frac{\omega-\mathbf{k} \cdot \mathbf{v}+\xi_{2}}{\xi_{d}}, \\
G_{2}(\mathbf{k}, \omega) & =\int d^{3} v F(\mathbf{v}) \frac{\omega-\mathbf{k} \cdot \mathbf{v}+\xi_{1}}{\xi_{d}} .
\end{aligned}
$$

Solving Eq.(25) for $\tilde{R}_{31}$ we obtain

$$
\tilde{R}_{31}(\mathbf{k}, \omega)=i \tilde{\Omega}_{1}(\mathbf{k}, \omega) \frac{n_{0}}{\gamma}\left(\frac{1-i \gamma G_{2}(\mathbf{k}, \omega)}{G_{d}(\mathbf{k}, \omega)}-1\right),
$$

where

$$
G_{d}(\mathbf{k}, \omega)=\left(1-i \gamma G_{1}\right)\left(1-i \gamma G_{2}\right)+\gamma^{2}\left|\Omega_{2}\right|^{2} G^{2} .
$$

We can now return to Eq.(21) and solve for $\tilde{\Omega}_{1}(\mathbf{k}, \omega)$. Since $\tilde{R}_{31}$ is linear in $\tilde{\Omega}_{1}$, we introduce the linear susceptibility by,

$$
\tilde{R}_{31}(\mathbf{k}, \omega)=\chi_{31}(\mathbf{k}, \omega) \frac{c}{g} \tilde{\Omega}_{1}(\mathbf{k}, \omega),
$$

and the complex wave-number,

$$
p(\mathbf{k}, \omega)=\frac{\omega}{c}-\frac{k^{2}}{2 q_{1}}+\chi_{31}(\mathbf{k}, \omega),
$$

to express Eq.(21) as

$$
\left[k_{z}-p(\mathbf{k}, \omega)\right] \tilde{\Omega}_{1}(\mathbf{k}, \omega)=0 .
$$

When the changes in the envelopes along the $z$-direction are much smaller compared to the changes in the transverse plane, we may replace $\mathbf{k} \rightarrow \mathbf{k}_{\perp}$ in the $\xi$ 's and the $G$ 's in Eqs.(24) and (26), where $\mathbf{k}_{\perp}$ is the projection of $\mathbf{k}$ onto the transverse plane, and write Eq. (31) in the $\left(z ; \mathbf{k}_{\perp}, \omega\right)$ coordinates:

$$
\frac{\partial}{\partial z} \tilde{\Omega}_{1}\left(z ; \mathbf{k}_{\perp}, \omega\right)=i p\left(\mathbf{k}_{\perp}, \omega\right) \tilde{\Omega}_{1}\left(z ; \mathbf{k}_{\perp}, \omega\right)
$$

or, the solution

$$
\tilde{\Omega}_{1}\left(z_{2} ; \mathbf{k}_{\perp}, \omega\right)=\tilde{\Omega}_{1}\left(z_{1} ; \mathbf{k}_{\perp}, \omega\right) e^{i p\left(\mathbf{k}_{\perp}, \omega\right)\left(z_{2}-z_{1}\right)} .
$$

Finally, the probe's envelope is given by

$$
\begin{aligned}
\tilde{\Omega}_{1}\left(z_{2} ; x, y, t\right) & =\int_{-\infty}^{\infty} \frac{d \omega}{2 \pi} e^{-i \omega t} \int_{-\infty}^{\infty} \frac{d^{2} k_{\perp}}{2 \pi} e^{i k_{x} x+i k_{y} y} \\
& \times \tilde{\Omega}_{1}\left(z_{1} ; \mathbf{k}_{\perp}, \omega\right) e^{i p\left(\mathbf{k}_{\perp}, \omega\right)\left(z_{2}-z_{1}\right)}
\end{aligned}
$$

\section{The Doppler-Dicke Transition}

It is first instructive to consider the case of ordinary one-photon absorption by taking $\Omega_{2}=0$, and to assume for simplicity a plane-wave stationary probe, namely $\omega=$ 0 and $\mathbf{k}=0$. For this case Eqs.(26)-(29) give

$$
\chi_{31}\left(\Delta_{1}\right)=i \frac{g n_{0}}{c} K\left(\Delta_{1}\right),
$$

where $K\left(\Delta_{1}\right)$ is the one-photon complex spectrum,

$$
K\left(\Delta_{1}\right)=\frac{i G_{1}\left(\Delta_{1}\right)}{1-i \gamma G_{1}\left(\Delta_{1}\right)},
$$

and $G_{1}\left(\Delta_{1}\right)$ takes the form of a Doppler-like profile (a Voigt convolution), with $\gamma$ being added to the standard homogenous-width, $\Gamma_{d}$ :

$$
G_{1}\left(\Delta_{1}\right)=\frac{1}{\sqrt{2 \pi} v_{\mathrm{th}}} \int d u \frac{e^{-u^{2} /\left(2 v_{\mathrm{th}}^{2}\right)}}{\Delta_{1}-q_{1} u+i\left(\Gamma_{d}+\gamma\right)}
$$

The spectrum $K\left(\Delta_{1}\right)$ in the form of Eq. (36) was previously presented by May [8] for one-photon transitions. Its extreme limits are [3, 8, 18]: the Doppler limit, trivially obtained by setting $\gamma=0$, and the Dicke limit, found for large $\gamma$. The Dicke parameter is $v_{\mathrm{th}} q_{1} / \gamma$, proportional to the ratio between the mean free-path and the radiation wavelength.

For optical transitions in room-temperature vapor, the one-photon line is usually in the far Doppler limit, i.e. $K \approx i G_{1}$. We have defined $K$ such that it is real for $\left|\Delta_{1}\right| \ll\left|q_{1} u+i\left(\Gamma_{d}+\gamma\right)\right|$, i.e. near the one-photon resonance where experiments in EIT are often done, and is equal to the on-resonance absorption (in frequency units). Specifically, for an atom at rest $(\gamma=0$ and no Doppler $), K\left(\Delta_{1}=0\right)=\Gamma_{d}^{-1}$.

A similar Doppler-Dicke transition occurs for the EIT transmission window. In order to demonstrate that, 


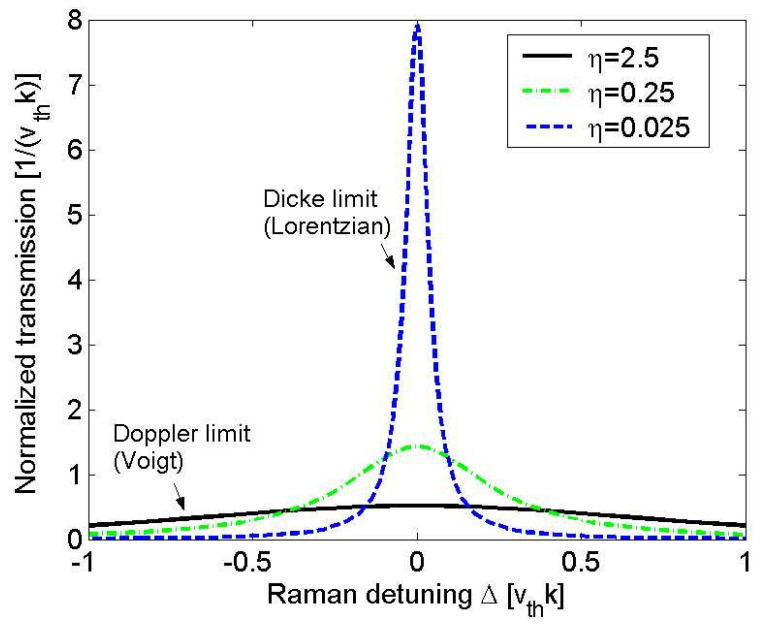

FIG. 3: Normalized EIT transmission spectra, numerically calculated from Eqs. (26)-(29), for three values of the EIT Dicke parameter, $\eta=v_{t h} k / \gamma$ (the ratio between the residual Doppler width and the velocity relaxation rate), with $\Gamma_{d}=2500 v_{t h} k, \Gamma_{21}=0.025 v_{t h} k$ and $\left|\Omega_{2}\right|^{2} / \Gamma_{d}=\Gamma_{21} / 25$ (small power-broadening). When $\eta$ is large (solid black line) the spectrum is a Voigt curve (a Gaussian-Lorentzian convolution). When $\eta$ is small (dashed blue line) the spectrum is a pure Lorentzian of width $\Gamma_{d}$. The dot-dashed green line demonstrates an intermediate result.

we have chosen a set of typical parameters with small power-broadening and calculated several EIT lines from Eqs.(26)-(29) by numerically integrating Eqs.(26). Three line shapes as a function of the normalized Raman detuning are presented in Figs. 3. The full-width at halfmaximum (FWHM), as a function of $|\mathbf{k}|$ and for various values of $\gamma$, is presented in Fig. 4. For the calculations we took a stationary $(\omega=0)$, collinear and degenerate $\left(\mathbf{q}_{1}=\mathbf{q}_{2}\right)$ EIT with $\mathbf{k} \perp \hat{\mathbf{z}}$, so that $k=|\mathbf{k}|$ is the wave-vector difference between the pump and the probe (similar results are obtained by replacing $k$ with $\left|\mathbf{q}_{1}-\mathbf{q}_{2}\right|$, when $\left.\left|\mathbf{q}_{1}-\mathbf{q}_{2}\right| \ll q_{1}\right)$. The residual Doppler width is expected to be $v_{\text {th }} k$, and the EIT-Dicke parameter is $\eta=v_{\text {th }} k / \gamma$ [18]. Fig. 4 clearly shows the transition between the linear (Doppler) regime, where $\eta \gg 1$, to the quadratic (Dicke) regime, where $\eta \ll 1$. The results of the numerical integrations throughout the Doppler-Dicke transition are well approximated (dashed lines in Fig. 4) by the analytic expression:

$$
\mathrm{FWHM}=2 \times \frac{2}{a^{2}} \gamma H\left(a \frac{v_{\mathrm{th}} k}{\gamma}\right)
$$

where $H(x)=e^{-x}-1+x$ and $a^{2}=2 / \ln 2$. The function $H(x)$ is usually associated with the velocity selfcorrelation in Brownian motion [18, 32], and its extreme limits are $H(x \rightarrow 0)=x^{2} / 2$ and $H(x \rightarrow \infty)=x$.

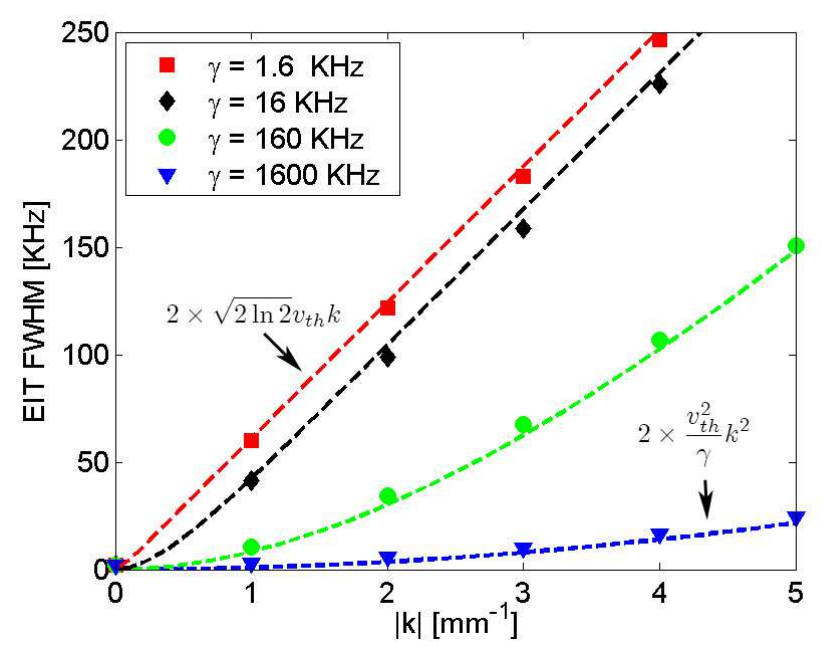

FIG. 4: Full width at half maximum of the EIT transparency window (points), obtained from numerical results similar to Fig. 3] as a function of the wave-vector difference, $k$, for various values of the velocity relaxation rate, $\gamma$. The dashed lines are given by Eq. (38). Other parameters are (typical for experiments with small power-broadening): $v_{t h}=170$ $\mathrm{m} / \mathrm{s}, \Gamma_{d}=100 \mathrm{MHz}, \Gamma_{21}=1 \mathrm{KHz},\left|\Omega_{2}\right|^{2} / \Gamma_{d}=40 \mathrm{~Hz}$. The three lines in Fig. 3 correspond here to $|k| \approx 1.5 \mathrm{~mm}^{-1}$ and $\gamma=16,160$ and $1600 \mathrm{KHz}$.

\section{THE DICKE-DIFFUSION SOLUTION}

In most realistic experiments with EIT, $\gamma$ is large enough to cause Dicke-narrowing of the two-photon line. In principle, this spectrum can be obtained by applying the large $\gamma$ limit to the general solution of the susceptibility of the system, Eqs. (27) and (29). However, since Eq.(27) is somewhat opaque, it seems worthwhile to first derive a diffusion-like equation for $R_{21}(\mathbf{r}, t)$, and then find the response of $R_{31}(\mathbf{r}, t)$. We do this first in the absence of fields and then in their presence, analyzing the cases of stationary and non-stationary probe.

\section{A. Diffusion during storage-of-light}

We are interested in describing the dynamics of the ground-state populations and coherences,

$$
R_{G}=\left(\begin{array}{ll}
R_{11} & R_{21}^{*} \\
R_{21} & R_{22}
\end{array}\right)
$$

in the absence of electromagnetic fields. This amounts to the situation investigated in recent storage-of-light experiments [30, 31], in which a probe beam with a non-trivial spatial envelope in the transverse plane was stored in an EIT vapor and then retrieved. Following Ref. [33], the storage procedure was described by a linear mapping of the probe field onto the ground-state coherence, $R_{21}(\mathbf{r}, t)$. It was then presumed that the dynamics "in 
the dark" can be described by the standard diffusion process, namely $\left[\dot{R}_{G}\right]_{\text {diffusion }}=D \nabla^{2} R_{G}$, where $D$ is the diffusion coefficient [30]. Eventually the retrieved probe field is obtained from $R_{21}(\mathbf{r}, t)$ by the reverse linear mapping.

The dynamics of $R_{21}(\mathbf{r}, t)$ can be described in terms of a diffusion-like equation. To this end we return to Eqs.(13), substitute $\Omega_{1}=\Omega_{2}=0$, and apply a procedure similar to that of Chapman-Enskog (see e.g. 34]). The equation for $\rho_{21}$ is

$$
\left[\frac{\partial}{\partial t}+\Gamma_{21}+\gamma+i \omega_{21}+\mathbf{v} \cdot \frac{\partial}{\partial \mathbf{r}}\right] \rho_{21}=\gamma R_{21}(\mathbf{r}, t) F(\mathbf{v})
$$

Integrating over velocity and using Eqs.(9) and (11), we have

$$
\left[\frac{\partial}{\partial t}+i\left(\omega_{21}-i \Gamma_{21}\right)\right] R_{21}(\mathbf{r}, t)+\frac{\partial}{\partial \mathbf{r}} \cdot \mathbf{J}_{21}(\mathbf{r}, t)=0,
$$

where

$$
\mathbf{J}_{s s^{\prime}}(\mathbf{r}, t)=\int d^{3} v \mathbf{v} \rho_{s s^{\prime}}(\mathbf{r}, \mathbf{v}, t)
$$

is the current density of the density-matrix. When $\gamma$ is dominant, we divide Eq. (40) by $\gamma$ and get, to zero order in $1 / \gamma$,

$$
\rho_{21}^{(0)}(\mathbf{r}, \mathbf{v}, t)=R_{21}(\mathbf{r}, t) F(\mathbf{v}),
$$

which carries no current. Thus, to find the current we go to first order in $1 / \gamma$,

$$
\rho_{21}(\mathbf{r}, \mathbf{v}, t)=R_{21}(\mathbf{r}, t) F(\mathbf{v})+\frac{1}{\gamma} \rho_{21}^{(1)}(\mathbf{r}, \mathbf{v}, t),
$$

substitute it into Eq.(40), multiply by $v_{j}$ and integrate over velocity,

$$
\begin{aligned}
& \int d^{3} v v_{j} {\left[v_{i} \frac{\partial}{\partial x_{i}}+\frac{\partial}{\partial t}+\Gamma_{21}+\gamma+i \omega_{21}\right] } \\
& \times\left(R_{21}(\mathbf{r}, t) F(\mathbf{v})+\frac{1}{\gamma} \rho_{21}^{(1)}(\mathbf{r}, \mathbf{v}, t)\right)=0 .
\end{aligned}
$$

Since

$$
\int d^{3} v v_{j} v_{i} \frac{\partial}{\partial x_{i}} R_{21}(\mathbf{r}, t) F(\mathbf{v})=\delta_{i j} v_{\mathrm{th}}^{2} \frac{\partial}{\partial x_{j}} R_{21}(\mathbf{r}, t),
$$

and

$$
\int d^{3} v v_{j} \rho_{21}^{(1)}(\mathbf{r}, \mathbf{v}, t)=\gamma J_{21, j}(\mathbf{r}, t)
$$

we find from Eq. (45), keeping only leading terms in $\gamma$,

$$
\mathbf{J}_{21}(\mathbf{r}, t)=-D \frac{\partial}{\partial \mathbf{r}} R_{21}(\mathbf{r}, t)
$$

where

$$
D=\frac{v_{\mathrm{th}}^{2}}{\gamma}
$$

is the spatial diffusion coefficient 32]. Substituting this result into Eq.(41), we find

$$
\frac{\partial}{\partial t} R_{21}(\mathbf{r}, t)=D \nabla^{2} R_{21}(\mathbf{r}, t)-\left(\Gamma_{21}+i \omega_{21}\right) R_{21}(\mathbf{r}, t)
$$

Eq. (50) describes a spatial diffusion of the coherence, accompanied by a homogenous decay of rate $\Gamma_{21}$ and a rotation of rate $\omega_{21}$. A similar derivation can be preformed for the ground-state populations, $R_{11}$ and $R_{22}$, and it results in a similar diffusion equation. This solution affirms the theoretical conjectures of Refs. [30, 31]. In what follows, it is generalized to describe the diffusion in the presence of the fields, i.e. during slow-light propagation.

It is interesting to note that even in the limit $\Gamma_{21} \rightarrow 0$, Eq.(50) results in the decay of the total stored-light energy. Assuming the stored coherence, $R_{21}$, is linear in the field's amplitude, the total intensity is proportional to the integral over $\left|R_{21}\right|^{2}$, which is not conserved and always decreases under diffusive spread. A similar observation was made in [35] in the context of stationary light pulses that diffuse along the $z$-direction (the diffusion equation therein originates from a different mechanism).

\section{B. Diffusion in the presence of fields}

Here we derive the dynamic equations for the envelopes of the densities, $\tilde{R}_{21}(\mathbf{r}, t)$ and $\tilde{R}_{31}(\mathbf{r}, t)$, along similar lines as above, while considering the interaction with the pump and the probe. For brevity, we omit the $(\mathbf{r}, t)$ notation and denote $\delta \mathbf{q}=\mathbf{q}_{1}-\mathbf{q}_{2}$. We start from the envelope equations, Eqs.(17), and integrate Eq.(17b) over velocity,

$$
\left(\frac{\partial}{\partial \mathbf{r}}+i \delta \mathbf{q}\right) \cdot \tilde{\mathbf{J}}_{21}+\left(\frac{\partial}{\partial t}-i \Delta+\Gamma_{21}\right) \tilde{R}_{21}=i \tilde{\Omega}_{2}^{*}(\mathbf{r}) \tilde{R}_{31}
$$

where $\tilde{\mathbf{J}}_{s s^{\prime}}$ is the envelope of the current densities, defined in analogy to Eq.(42). We expand $\tilde{\rho}_{21}$ as in Eq.(44), $\tilde{\rho}_{21}=\tilde{R}_{21} F(\mathbf{v})+(1 / \gamma) \tilde{\rho}_{21}^{(1)}$, multiply Eq. $117 \mathrm{~b}$ by $\mathbf{v}$ and integrate over velocity. Using Eqs.(46) and (47), and keeping leading terms in $\gamma$, we find

$$
\tilde{\mathbf{J}}_{21}+D\left(\frac{\partial}{\partial \mathbf{r}}+i \delta \mathbf{q}\right) \tilde{R}_{21}=i \frac{\tilde{\Omega}_{2}^{*}(\mathbf{r})}{\gamma} \tilde{\mathbf{J}}_{31},
$$

where $D$ is defined in Eq.(49). Substituting $\tilde{\mathbf{J}}_{21}$ back into Eq. (51) we get

$$
\begin{aligned}
& {\left[\frac{\partial}{\partial t}-i \Delta+\Gamma_{21}-D\left(\frac{\partial}{\partial \mathbf{r}}+i \delta \mathbf{q}\right)^{2}\right] \tilde{R}_{21}} \\
& =i \tilde{\Omega}_{2}^{*}(\mathbf{r}) \tilde{R}_{31}-i\left(\frac{\partial}{\partial \mathbf{r}}+i \delta \mathbf{q}\right) \cdot \frac{\tilde{\Omega}_{2}^{*}(\mathbf{r})}{\gamma} \tilde{\mathbf{J}}_{31} .
\end{aligned}
$$


In order to calculate $\tilde{R}_{31}$ and $\tilde{\mathbf{J}}_{31}$, we assume in Eq.(17a) that temporal and spatial changes in the envelope of the probe are much smaller then the one-photon homogenous decoherence rate $\left(\Gamma_{d}+\gamma\right)$ and the wavenumber $\left(q_{1}\right)$, respectively:

$$
\left|\frac{\partial}{\partial t}+\mathbf{v} \cdot \frac{\partial}{\partial \mathbf{r}}\right| \ll\left|\xi_{1}\right|=\left|\Delta_{1}-\mathbf{q}_{1} \cdot \mathbf{v}+i\left(\Gamma_{d}+\gamma\right)\right| .
$$

We then formally solve Eq.117a) for $\tilde{\rho}_{31}$ and substitute only the dominant part of $\tilde{\rho}_{21}$, i.e. $\tilde{\rho}_{21}^{(0)}=\tilde{R}_{21} F(\mathbf{v})$, to find

$$
\tilde{\rho}_{31}(\mathbf{r}, \mathbf{v}, t)=\frac{\left[i \gamma \tilde{R}_{31}-\tilde{\Omega}_{2}(\mathbf{r}) \tilde{R}_{21}-\tilde{\Omega}_{1}(\mathbf{r}, t) n_{0}\right] F(\mathbf{v})}{\left(\Delta_{1}-\mathbf{q}_{1} \cdot \mathbf{v}+i\left(\Gamma_{d}+\gamma\right)\right)} .
$$

Integrating Eq.(55) over velocity, we get

$$
\tilde{R}_{31}=G_{1}\left[i \gamma \tilde{R}_{31}-\tilde{\Omega}_{2}(\mathbf{r}) \tilde{R}_{21}-\tilde{\Omega}_{1}(\mathbf{r}, t) n_{0}\right],
$$

where $G_{1}$ of Eq.(37) is the Doppler profile, or

$$
\tilde{R}_{31}(\mathbf{r}, t)=i K\left[\tilde{\Omega}_{1}(\mathbf{r}, t) n_{0}+\tilde{\Omega}_{2}(\mathbf{r}) \tilde{R}_{21}(\mathbf{r}, t)\right],
$$

where $K=K\left(\Delta_{1}\right)$ of Eq.(36) is the one-photon absorption spectrum, which in the Dicke limit of the EIT can be considered as a constant near the EIT line.

Eq.(53) and Eq.(57) form a complete set for $\tilde{R}_{21}$ and $\tilde{R}_{31}$, only when the term

$$
\left(\frac{\partial}{\partial \mathbf{r}}+i \delta \mathbf{q}\right) \cdot \frac{\tilde{\Omega}_{2}^{*}(\mathbf{r})}{\gamma} \tilde{\mathbf{J}}_{31}
$$

in Eq.(53) can be neglected. This term vanishes completely in the special case of pump and probe which are plane-waves $(\partial / \partial \mathbf{r}=0)$, collinear and degenerate $(\delta \mathbf{q}=$ $0)$. It can also be neglected whenever $\left|\Omega_{2}\right| \ll \gamma$, as is the case in many realistic situations, cf. [21]. Furthermore, if the latter condition is not satisfied, we can still neglect the term (58) when both the spatial variations $(\partial / \partial \mathbf{r})$ and $\delta \mathbf{q}$ reside in the transverse plane, perpendicular to $\mathbf{q}_{1}$, since $\tilde{\mathbf{J}}_{31} \| \mathbf{q}_{1}$ (as can be found by multiplying Eq.(55) by $\mathbf{v}$ and integrating). Discarding this term from Eq.(53), and together with Eq.(57), we find for $\tilde{R}_{21}$ a diffusion-like equation,

$$
\begin{aligned}
& {\left[\frac{\partial}{\partial t}-i \Delta+\Gamma_{21}+K\left|\tilde{\Omega}_{2}(\mathbf{r})\right|^{2}\right] \tilde{R}_{21}(\mathbf{r}, t)} \\
& =D\left(\frac{\partial}{\partial \mathbf{r}}+i \delta \mathbf{q}\right)^{2} \tilde{R}_{21}(\mathbf{r}, t)-n_{0} K \tilde{\Omega}_{2}^{*}(\mathbf{r}) \tilde{\Omega}_{1}(\mathbf{r}, t) .
\end{aligned}
$$

Eq.(59) is the extension of Eq.(50) in the presence of fields, and it is written in terms of the envelopes. The term $i \delta \mathbf{q}$ is responsible for the diffusion across the fields' interference pattern, created when $\mathbf{q}_{1} \neq \mathbf{q}_{2}$. Once we solve the diffusion equation for $\tilde{R}_{21}(\mathbf{r}, t)$ we substitute it in Eq.(57) and obtain $\tilde{R}_{31}(\mathbf{r}, t)$. We carry this out in the next subsection for the case of a plane-wave pump and in section $\nabla$ for a finite-size pump.

\section{The Dicke-like Absorption Spectrum}

We would like to calculate the susceptibility of the system for the case of a plane-wave pump, $\tilde{\Omega}_{2}(\mathbf{r})=\Omega_{2}$. We Fourier transform in $\mathbf{r}$ and $t$ using Eq.(19) and turn Eq.(59) into

$$
\begin{aligned}
& {\left[i(\Delta+\omega)-\Gamma_{21}-K\left|\Omega_{2}\right|^{2}\right] \tilde{R}_{21}(\mathbf{k}, \omega)} \\
& =D(\delta \mathbf{q}+\mathbf{k})^{2} \tilde{R}_{21}(\mathbf{k}, \omega)+n_{0} K \Omega_{2}^{*} \tilde{\Omega}_{1}(\mathbf{k}, \omega)
\end{aligned}
$$

and Eq.(57) into

$$
\tilde{R}_{31}(\mathbf{k}, \omega)=i K\left[\tilde{\Omega}_{1}(\mathbf{k}, \omega) n_{0}+\Omega_{2} \tilde{R}_{21}(\mathbf{k}, \omega)\right] .
$$

Solving Eq.(60) for $\tilde{R}_{21}(\mathbf{k}, \omega)$, substituting in Eq.(61) to obtain $\tilde{R}_{31}$ and using Eq.(29), we find the susceptibility in the diffusion-limit case to be

$$
\chi_{31}(\mathbf{k}, \omega)=\frac{g}{c} i K n_{0}[1-L(\mathbf{k}, \omega)],
$$

where

$$
L(\mathbf{k}, \omega)=\frac{-K\left|\Omega_{2}\right|^{2}}{i(\Delta+\omega)-\Gamma_{\mathrm{hom}}-D(\delta \mathbf{q}+\mathbf{k})^{2}},
$$

and

$$
\Gamma_{\text {hom }}=\Gamma_{21}+K\left|\Omega_{2}\right|^{2}
$$

is the EIT width in the absence of diffusion.

The absorption of the probe is proportional to $\Im m \chi_{31}$. As explained in section [II, in typical cases $K$ is real and therefore

$$
\Im m \chi_{31}=\frac{g}{c} K n_{0}[1-\Re e L(\mathbf{k}, \omega)],
$$

i.e. the well-known EIT absorption spectrum is composed of the one-photon absorption, $g K n_{0} / c$, and a "transparency window", $\Re e L(\mathbf{k}, \omega)$, of Lorentzian shape:

$$
\Re e L(\mathbf{k}, \omega)=\frac{K\left|\Omega_{2}\right|^{2}\left[\Gamma_{\mathrm{hom}}+D(\delta \mathbf{q}+\mathbf{k})^{2}\right]}{(\Delta+\omega)^{2}+\left[\Gamma_{\mathrm{hom}}+D(\delta \mathbf{q}+\mathbf{k})^{2}\right]^{2}} .
$$

Considering $L(\mathbf{k}, \omega)$ for a given $\mathbf{k}$, as a function of the Raman detuning $(\Delta+\omega)$, we find the homogenous EIT width to be $\Gamma_{\text {hom }}=\Gamma_{21}+K\left|\Omega_{2}\right|^{2}$, broadened by the Dicke-EIT width, $D\left(\mathbf{q}_{1}-\mathbf{q}_{2}+\mathbf{k}\right)^{2}$. Eqs. (65) and (66) generalize the results of our previous work in Ref. 18] for a finite probe in space and time. It includes the powerbroadening effect, $K\left|\Omega_{2}\right|^{2}$ that was absent in Ref.[18], in which the low-contrast approximation was taken. Notice that the term $K\left|\Omega_{2}\right|^{2}$ replaces the standard powerbroadening term, $\left|\Omega_{2}\right|^{2} / \Gamma_{d}$, to incorporate the Dopplerbroadening of the one-photon line. 


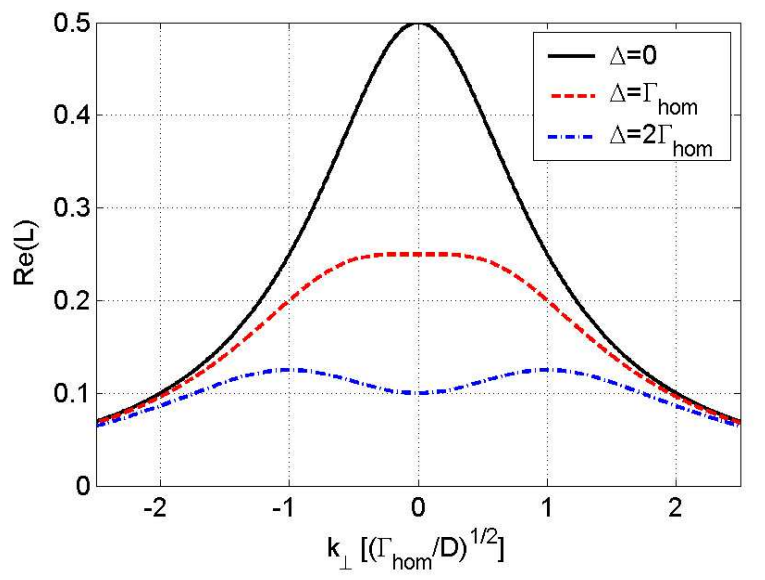

FIG. 5: The spatial-frequency filter (normalized EIT transmission), given in Eq. (66), as a function of $\left|\mathbf{k}_{\perp}\right|=|k \hat{\mathbf{x}}|$, for different Raman detunings, with $\omega=0, \delta \mathbf{q}=0$ and $\Gamma_{21}=$ $K\left|\Omega_{2}\right|^{2}$. The solid-black curve is plotted for $\Delta=0$. The dashed red and blue curves demonstrate the effect of nonzero Raman detuning: a decrease in transparency alongside a change in the curvature near $k=0$.

\section{Spatial-frequency filter and diffusion-like behavior}

When $\Im m \chi_{31}(\mathbf{k}, \omega)$ is considered in steady-state $(\omega=$ $0)$ as a function of $\mathbf{k}$, it acts as a spatial-frequency filter for the probe beam. We have in mind a stationary probe beam in the plane $z=z_{1}$ with an envelope $\tilde{\Omega}_{1}\left(z_{1} ; x, y\right)$, which propagates through the medium to the $z=z_{2}$ plane. Following Eq.(34), $\Im m \chi_{31}\left(\mathbf{k}_{\perp}, \omega\right)$ determines the absorption of each spatial-frequency component of $\tilde{\Omega}_{1}\left(z ; \mathbf{k}_{\perp}\right)$. Since the first term in Eq. (65) - the one-photon absorption - is constant for all $\mathbf{k}_{\perp}$, the filter becomes a "transmission" filter with the shape $\Re e L\left(\mathbf{k}_{\perp}\right)$. Several examples for this filter are plotted in Fig. 5 with $\Gamma_{\text {hom }}=2 \Gamma_{12}$ and $\delta \mathbf{q}=0$. On Raman-resonance, the curve is a Lorentzian of width $k_{\text {typ }}=\sqrt{\Gamma_{\text {hom }} / D}$, and the maximum overall transmission is achieved. For nonzero Raman detuning it obtains a more detailed structure - the curvature at $k=0$ decreases, becomes zero for $\Delta=\Gamma_{\text {hom }}$, and turns negative for $\Delta>\Gamma_{\text {hom }}$.

Figure 6 presents possible outcomes of $\tilde{\Omega}_{1}\left(z_{2} ; x, y\right)$, for several $\tilde{\Omega}_{1}\left(z_{1} ; x, y\right)$ (representing the absolute value of $\tilde{\Omega}_{1}$ as two-dimensional images). When the features in the incident image are large, namely when $\tilde{\Omega}_{1}\left(z_{1} ; \mathbf{k}_{\perp}\right)$ is confined within $k_{\perp} \ll k_{\text {typ }}$, the filter is approximately quadratic in $k_{\perp}$ (the central part of the solid-black line in Fig. (5), which results in pure diffusion in real space. This is demonstrated in Figs. 6(a) and 6(b), where the featuresize is of the order of $2 \pi / k_{\text {typ }}$. In Fig. 6(c) we demonstrate the property of complex diffusion - the phase of the left line in $\tilde{\Omega}_{1}\left(z_{1} ; x, y\right)$ was shifted by $\pi$, causing a destructive interference between atoms that diffuse to

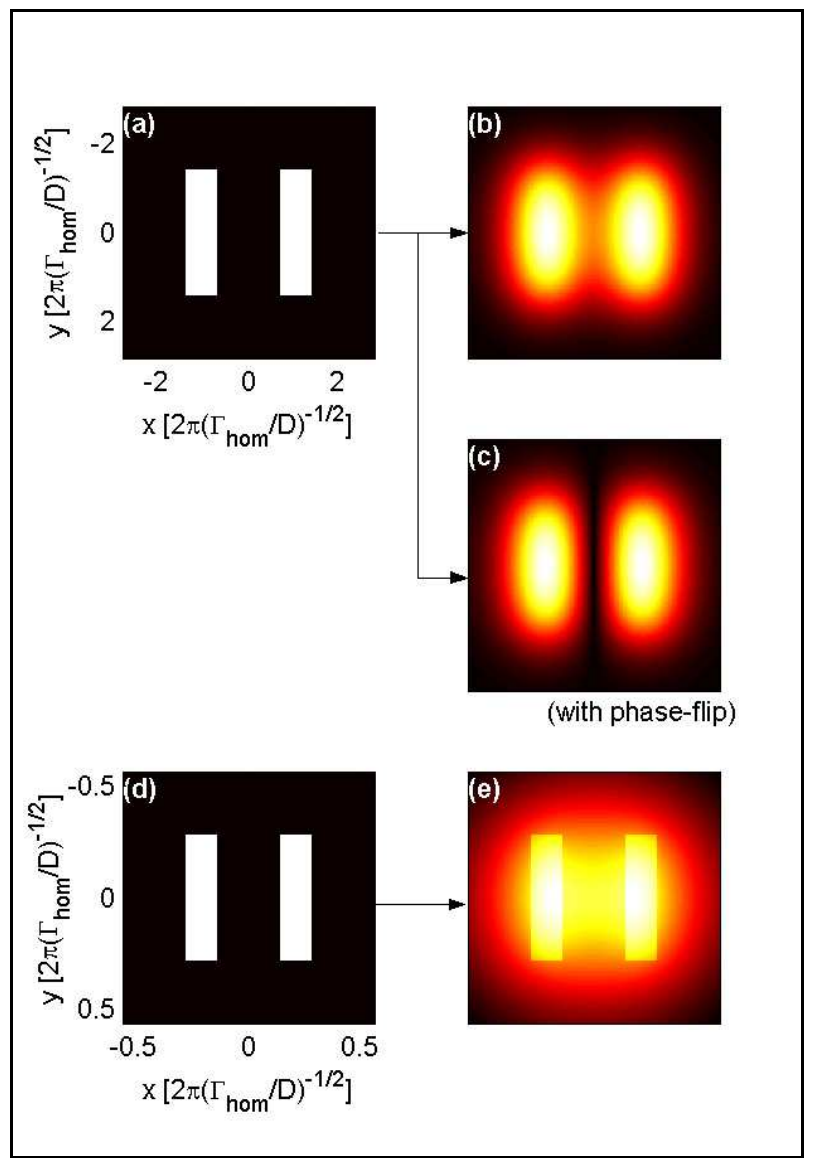

FIG. 6: Calculated effect of the spatial-frequency filter with a plane-wave pump and a finite probe beam: The initial pattern, $\left|\tilde{\Omega}_{1}\left(z_{1} ; x, y\right)\right|$ (left), and the transmitted pattern, $\left|\tilde{\Omega}_{1}\left(z_{2} ; x, y\right)\right|$, after a certain propagation length (right). The calculations were done using Eqs. (30), (34), (62) and (63), and the diffraction $\left(k^{2} / 2 / q_{1}\right)$ was neglected for clarity. The parameters correspond to the black line in Fig. $5\left(K\left|\Omega_{2}\right|^{2}=\right.$ $\left.\Gamma_{12}, \delta q=0, \Delta=0\right)$. Images (a)-(c) illustrate regular diffusion of real (b) and complex (c) fields. To generate (c), the phase of the left line in the incident field was flipped. Images (d) and (e) illustrate the effect for smaller features, when larger $k_{\perp}$ 's are pronounce.

the area between the lines, keeping it dark indefinitely 31]. When smaller features exist and $k_{\perp}$ extends beyond $k_{\text {typ }}$ a more elaborate behavior occurs. Figure 6(d) is a small-scale version of 6(a) and we see, by comparing 6(e) and 6(b), that the Lorentzian-shaped filter preserves the sharp edges in the smaller image. This is due to the substantial deviation of the Lorentzian filter from a quadratic-shaped filter (pure diffusion) for higher $k$ 's.

A direct measurement of the filter was carried out in Refs. [20, 21]. In these experiments, the transmission of a stationary $(\omega=0)$ on-resonance $(\Delta=0)$ probe beam was measured in two cases: an almost planewave probe, propagating with a small angular deviation from the pump $(\delta \mathbf{q} \neq 0 ; \mathbf{k}=0)$, and a divergent probe $(\delta \mathbf{q}=0 ; \mathbf{k} \neq \mathbf{0})$. In both cases, the wave-number was 
written as a function of the angular deviation $(\theta)$, as $|\delta \mathbf{q}+\mathbf{k}|=q \theta$, and the measured transmission agreed with

$$
\Re e L(\mathbf{k})=\frac{K\left|\Omega_{2}\right|^{2}}{\Gamma_{\text {hom }}+D q^{2} \theta^{2}} .
$$

We observe that, in the diverging beam experiment [21], the outer parts of the beam are absorbed and consequently the beam radius decreases. This result is counterintuitive from the viewpoint of diffusion, since diffusion usually results in spreading, rather than contraction, of an initial profile. Nevertheless, in the process of complex diffusion, a reduction in size can occur through destructive interference - the diffusion wipes out the field in regions where the phase pattern exhibits rapid changes. The relative phase between a diverging Gaussian probe beam and a plane-wave pump beam exhibits rapid variations in the transverse direction as the axial distance increases, and thus diffusion results in a gradual elimination of the outer parts of the beam.

\section{E. Diffusion of slow-light}

To conclude this section, we analyze the non-stationary behavior of slow-light propagation. We consider the case of colinear pump and probe, $\mathbf{q}_{1}\left\|\mathbf{q}_{2}\right\| \hat{\mathbf{z}}$, and assume that the changes in the probe's envelope along $z$ are much smaller than the changes in the transverse plane, such that $\delta \mathbf{q} \cdot \mathbf{k} \ll k^{2}$ (or alternatively, take $\delta \mathbf{q}=0$ ). Denoting $\mathbf{k}=\mathbf{k}_{\perp}+k_{z} \hat{\mathbf{z}}$, with $k_{z} \ll k_{\perp}$, Eq. (63) becomes

$$
L=\frac{-K\left|\Omega_{2}\right|^{2}}{i(\Delta+\omega)-\Gamma_{\mathrm{hom}}-D \delta q^{2}-D k_{\perp}^{2}} .
$$

We further take the standard slow-light assumption, that the band-width of the probe pulse is fully within the linear dispersion regime, i.e. that $|\Delta+\omega| \ll\left|\Gamma_{\text {hom }}+D \delta q^{2}\right|$. More importantly, we assume that $D k_{\perp}^{2} \ll \Gamma_{\text {hom }}+D \delta q^{2}$, i.e. that the correction to the EIT width resulting from the finite size of the probe is much smaller than the EIT width of a plane-wave probe. The latter condition prevents the dispersion of different spatial-frequency components of the envelope, and is the essence of the diffusion approximation, allowing us to write $L$ as quadratic in $k_{\perp}^{2}$,

$$
L \approx \frac{K\left|\Omega_{2}\right|^{2}}{\Gamma_{\mathrm{hom}}+D \delta q^{2}}\left(1+\frac{i(\Delta+\omega)-D k_{\perp}^{2}}{\Gamma_{\mathrm{hom}}+D \delta q^{2}}\right) .
$$

We return to the envelope equations of the probe, Eqs.(30)-(31), and use the susceptibility of Eq.(62),

$$
\left[k_{z}-\frac{\omega}{c}+\frac{k_{\perp}^{2}}{2 q_{1}}-i \frac{g}{c} K n_{0}(1-L)\right] \tilde{\Omega}_{1}(\mathbf{k}, \omega)=0 .
$$

Substituting $L$ and defining the group-velocity $V_{g}$ as

$$
\frac{c}{V_{g}}=1+\frac{g n_{0} K^{2}\left|\Omega_{2}\right|^{2}}{\left(\Gamma_{\mathrm{hom}}+D \delta q^{2}\right)^{2}}
$$

we obtain

$$
\begin{aligned}
& {\left[i k_{z}-\frac{i \omega}{V_{g}}+\frac{i k_{\perp}^{2}}{2 q_{1}}+\frac{g n_{0}}{c} K-\right.} \\
& \left.\left(\frac{1}{V_{g}}-\frac{1}{c}\right)\left(\Gamma_{\text {hom }}+D \delta q^{2}-D k_{\perp}^{2}+i \Delta\right)\right] \tilde{\Omega}_{1}(\mathbf{k}, \omega)=0 .
\end{aligned}
$$

Returning to the time and space coordinates and assuming $V_{g} \ll c$ for brevity, we find

$$
\left[V_{g} \frac{\partial}{\partial z}+\frac{\partial}{\partial t}-\left(i \frac{V_{g}}{2 q_{1}}+D\right) \nabla_{\perp}^{2}+\Gamma_{0}-i \Delta\right] \tilde{\Omega}_{1}(\mathbf{r}, t)=0,
$$

where $\Gamma_{0}=V_{g} g n_{0} K / c-\Gamma_{\text {hom }}-D \delta q^{2}$ is the on-resonance decay rate and $\nabla_{\perp}^{2}$ is the Laplacian perpendicular to the $z$-axis. Introducing the traveling envelope of the probe beam, $\tilde{\Omega}_{1}^{\text {trav }}(\mathbf{r}, t)$, as

$$
\tilde{\Omega}_{1}(\mathbf{r}, t)=\tilde{\Omega}_{1}^{\operatorname{trav}}\left(\mathbf{r}-\hat{\mathbf{z}} V_{g} t, t\right) e^{\left(i \Delta-\Gamma_{0}\right) t},
$$

we find that it undergoes a simple diffusion equation with a non-real coefficient:

$$
\frac{\partial}{\partial t} \tilde{\Omega}_{1}^{\text {trav }}(\mathbf{r}, t)=\left(i \frac{V_{g}}{2 q_{1}}+D\right) \nabla_{\perp}^{2} \tilde{\Omega}_{1}^{\operatorname{trav}}(\mathbf{r}, t) .
$$

According to Eq. (75), a probe field with an arbitrary complex envelope that satisfies the slow-light assumptions will undergo both diffusion, as a result of the atomic thermal motion, and optical diffraction. The diffraction depends on the actual distance traveled by the beam (due to the factor $V_{g} / c$ ), while the diffusion depends on the time duration.

An interesting and important example is the propagation of a paraxial Gaussian beam, such as a HermiteGaussian or a Laguerre-Gauss mode [36]. These modes have the well-known property of being self-similar under diffraction, i.e. during the propagation through a diffractive medium their transverse shape remains unchanged up to a length-scale factor. It can be shown from Eq.(75), by utilizing the "complex scaling factor" representation 36], that Gaussian modes are also self-similar under diffusion. For example, the lowest order mode has a Gaussian intensity profile, which is known to maintain a Gaussian shape when diffusing. It can further be shown from Eq. (75) that the Gaussian modes experience a diffusion-induced decay throughout the propagation, as discussed at the end of $\S \S \mathrm{IVA}$.

\section{FINITE PUMP AND PROBE BEAMS}

When both the probe and the pump beams are finite, atoms can leave the light beams, evolve "in the dark" and diffuse back inside. It was recently demonstrated that such a process may result in an EIT line much narrower than expected from time-of-flight (TOF) broadening and power broadening - a phenomenon denoted as Ramsey narrowing [24]. The line shapes resulting from TOF broadening and Ramsey narrowing 
can be described by following the possible atomic paths (trajectories), calculating temporal probability functions for the atoms' location, and averaging over them (cf. [24]). If the time it takes to achieve steady-state with the driving field (pumping rate) is comparable to the TOF, it is commonly claimed that calculations cannot be done with standard steady-state approaches and timedependent solutions have to be used [37]. Nevertheless, here we calculate these effects using the steady-state solution of the diffusion equation in the presence of the fields. This is an exact and easier approach that allows more elaborate beam geometries to be considered. Note that the Ramsey-narrowing experiments are usually done with equal pump and probe intensities, while our model is for the weak probe regime. However, we expect the main attributes of the spectrum to be essentially the same for both cases.

We consider finite probe and pump beams and restrict the discussion to a colinear EIT, $\mathbf{q}_{1}-\mathbf{q}_{2}=\delta q \hat{\mathbf{z}}$. We assume that the fields are stationary and overlap in their cross sections with a neglected variation along the $z$-direction,

$$
\tilde{\Omega}_{1}(\mathbf{r}, t)=\Omega_{1} w\left(\mathbf{r}_{\perp}\right) \quad ; \quad \tilde{\Omega}_{2}(\mathbf{r})=\Omega_{2} w\left(\mathbf{r}_{\perp}\right),
$$

with $w\left(\mathbf{r}_{\perp}\right)$ the transverse profile of the fields. In the diffusion regime we use Eqs.(59) and (57), which can now be written as

$$
\begin{aligned}
& {\left[\Gamma-i \Delta+K\left|\Omega_{2}\right|^{2}\left|w\left(\mathbf{r}_{\perp}\right)\right|^{2}-D \nabla_{\perp}^{2}\right] \tilde{R}_{21}\left(\mathbf{r}_{\perp}\right)=} \\
& -n_{0} K \Omega_{2}^{*} \Omega_{1}\left|w\left(\mathbf{r}_{\perp}\right)\right|^{2},
\end{aligned}
$$

and

$$
\tilde{R}_{31}\left(\mathbf{r}_{\perp}\right)=i K\left[\Omega_{2} \tilde{R}_{21}\left(\mathbf{r}_{\perp}\right)+\Omega_{1} n_{0}\right] w\left(\mathbf{r}_{\perp}\right),
$$

where we denoted $\Gamma=\Gamma_{21}+D \delta q^{2}$ to be the non-powerbroadened width. In what follows, we solve for $\tilde{R}_{21}\left(\mathbf{r}_{\perp}\right)$ and $\tilde{R}_{31}\left(\mathbf{r}_{\perp}\right)$ in a specific example and calculate the resulting absorption spectrum.

\section{Example: a Stepwise Beam}

We consider a probe and a pump beams with uniform intensity and phase within a sheet of thickness $2 a$ in the $x$-direction (one-dimensional stepwise beams):

$$
w(x, y)= \begin{cases}1 & \text { for }|x| \leq a \\ 0 & \text { for }|x|>a\end{cases}
$$

Eq.(77) can then be written as

$$
\begin{aligned}
& D\left(k_{1}^{2}-\frac{\partial^{2}}{\partial x^{2}}\right) \tilde{R}_{21}(|x| \leq a)=-n_{0} K \Omega_{2}^{*} \Omega_{1}, \\
& D\left(k_{2}^{2}-\frac{\partial^{2}}{\partial x^{2}}\right) \tilde{R}_{21}(|x|>a)=0
\end{aligned}
$$

where

$$
\begin{aligned}
& k_{1}=\sqrt{\left(\Gamma+K\left|\Omega_{2}\right|^{2}-i \Delta\right) / D}, \\
& k_{2}=\sqrt{(\Gamma-i \Delta) / D},
\end{aligned}
$$

and $\Re e\left\{k_{i}\right\}>0$. For $|x| \leq a$ we expect a solution symmetric in $x$ and for $|x|>a$ we expect a solution decaying for $|x| \rightarrow \infty$. We thus find

$$
\begin{aligned}
& \tilde{R}_{21}(|x| \leq a)=A \cosh \left(k_{1} x\right)-\frac{K n_{0}}{k_{1}^{2} D} \Omega_{2}^{*} \Omega_{1}, \\
& \tilde{R}_{21}(|x|>a)=B \exp \left[-k_{2}(|x|-a)\right],
\end{aligned}
$$

and the coefficients $A$ and $B$ are obtained from the continuity conditions of $\tilde{R}_{21}$ and $\frac{\partial}{\partial x} \tilde{R}_{21}$ at $|x|=a$ :

$$
\begin{aligned}
A & =\frac{K n_{0}}{k_{1}^{2} D} \frac{\Omega_{2}^{*} \Omega_{1}}{\cosh \left(k_{1} a\right)+\left(k_{1} / k_{2}\right) \sinh \left(k_{1} a\right)}, \\
B & =A \cosh \left(k_{1} a\right)-\frac{K n_{0}}{k_{1}^{2} D} \Omega_{2}^{*} \Omega_{1} .
\end{aligned}
$$

For $\tilde{R}_{31}(x)$ we find from Eq.(78) that $\tilde{R}_{31}(|x|>a)=0$ and

$$
\begin{aligned}
\tilde{R}_{31}(|x|<a) & =i \Omega_{1} n_{0} K\left(1-\frac{K\left|\Omega_{2}\right|^{2}}{k_{1}^{2} D}\right) \\
& +i K A \Omega_{2} \cosh \left(k_{1} x\right)
\end{aligned}
$$

The energy absorption at frequency $\omega_{1}$ is

$$
P(\Delta)=2 \hbar \omega_{1} \frac{1}{2 a} \int_{-a}^{a} d x \Im m\left\{\Omega_{1}^{*} \tilde{R}_{31}(x)\right\}
$$

and we find

$$
P(\Delta)=P_{0} \Re e\left\{K-\frac{K^{2}\left|\Omega_{2}\right|^{2}}{\Gamma+K\left|\Omega_{2}\right|^{2}-i \Delta}\left[1-S_{D}(\Delta)\right]\right\},
$$

where $P_{0}=2 \hbar \omega_{1} n_{0}\left|\Omega_{1}\right|^{2}$ and

$$
S_{D}(\Delta)=\frac{\tanh \left(k_{1} a\right)}{k_{1} a} \frac{1}{1+\left(k_{1} / k_{2}\right) \tanh \left(k_{1} a\right)}
$$

is the correction resulting from the finite size of the beam. Figure 7 depicts $P(\Delta)$ and $\Re e S_{D}(\Delta)$ for $a=100 \mu \mathrm{m}$ and $a \rightarrow \infty$ (plane-wave) with typical parameters. The outer part of the finite-beam spectrum (dashed-blue) is broadened due to the TOF effect. Ramsey-narrowing is apparent in the central part as a cusp-like curve. The cusp is narrower than the power-broadened Lorentzian, but it is nevertheless limited by the width $\Gamma=\Gamma_{21}+$ $D \delta q^{2}$.

A similar calculation can be done for the twodimensional analogue of the stepwise sheet: a stepwise cylindrical profile with $w(r \leq a)=1$ and $w(r>a)=0$, where $r^{2}=x^{2}+y^{2}$. Solving Eqs. (77) and (78) in cylindrical symmetry, one finds that the energy absorption 


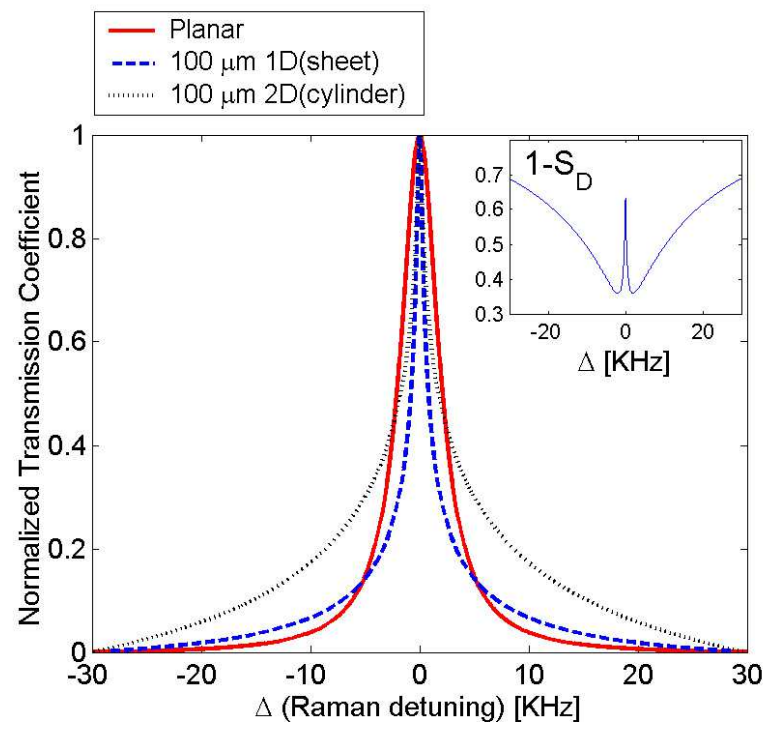

FIG. 7: Normalized transmission for a plane-wave and a finite-sized beam (1D and 2D), demonstrating TOF broadening and Ramsey-narrowing. The inset depicts the correction to the spectrum, resulting from the finiteness of beams, $\Re e\left[1-S_{D}(\Delta)\right]$, for the 1D case [Eq.(87)]. The parameters are: $\Gamma=100 \mathrm{~Hz}, K\left|\Omega_{2}\right|^{2}=2 \mathrm{KHz}, D=10 \mathrm{~cm}^{2} / \mathrm{sec}$ and $a=100 \mu \mathrm{m}$. The choice of $K\left|\Omega_{2}\right|^{2} \gg \Gamma$ makes the narrowing effect more obvious.

spectrum, $P(\Delta)$, has the exact same form of Eq.86), with the correction term being

$$
S_{D}(\Delta)=\frac{2}{k_{1} a}\left[\frac{I_{0}\left(k_{1} a\right)}{I_{1}\left(k_{1} a\right)}+\frac{k_{1}}{k_{2}} \frac{K_{0}\left(k_{2} a\right)}{K_{1}\left(k_{2} a\right)}\right]^{-1} .
$$

Here, $k_{1,2}$ are as defined in Eqs.(81) and $I_{0}(x)$ and $K_{0}(x)$ are modified Bessel functions. A comparison between the $1 \mathrm{D}$ and the $2 \mathrm{D}$ spectra is given in Fig. 7. In the $2 \mathrm{D}$ case the TOF effect is substantial while the Ramseynarrowing is reduced. The latter can be attributed to the fact that on average less atoms return to the beam in the $2 \mathrm{D}$ geometry.

\section{CONCLUSIONS}

We have presented a model for EIT that incorporates thermal atomic motion by introducing the density-matrix distribution in space and velocity along with a Boltzmann relaxation term. The model describes a range of motional phenomena, such as Dicke narrowing, Ramsey narrowing and diffusion during storage of light, which have been analyzed in the past in different independent studies. In the absence of electromagnetic fields, the model reduces to pure diffusion of the ground-state coherence and population, in agreement with recent storage of light experiments. In the presence of a pump and a weak probe fields, the solution for the spatial distribution of the atomic coherence and the fields can be obtained.
For the case of a plane-wave pump, the temporal and spatial frequency components of the incoming probe beam form the natural basis for the problem. The general solution, in terms of the complex susceptibility of the medium, $\chi_{31}(\mathbf{k}, \omega)$, exhibits the Doppler-Dicke transition for both the one-photon and the two-photon absorption spectra. For the realistic regime, in the presence of a buffer-gas, when the one-photon line is Dopplerbroadened and the two-photon line is Dicke-narrowed, an explicit expression for the EIT transmission, $L(\mathbf{k}, \omega)$, is derived. $L(\mathbf{k}, \omega)$ yields the EIT absorption spectrum, for any given $\mathbf{k}$, including the power-broadening effect and the Dicke width. Moreover, for any given $\omega, L(\mathbf{k}, \omega)$ serves as a spatial-frequency filter, generally diminishing high $k$ values. We explain this diminution by the diffusion of atoms across the pump-probe interference pattern, of wave-vector $\mathbf{k}+\mathbf{q}_{1}-\mathbf{q}_{2}$. On Raman-resonance, and when the spatial features of the incoming probe beam are large, the probe's envelope undergoes a diffusion-like dynamics. For smaller features or for non-zero Ramandetuning, a more elaborate behavior takes place. We note that a unique result is obtained for probe beams with a single value of $\left|\mathbf{k}_{\perp}\right|$, usually referred to as non-diffracting, e.g. Bessel beams [38]. These will not be distorted by $L(\mathbf{k}, \omega)$ and hence will not spread due to neither diffusion nor diffraction.

Since the complex amplitude of the probe's envelope diffuses, interference phenomena occur. For example, destructive interference between adjacent features that are opposite in phase, maintains the dark area between them. This also explains why adjacent rings in the Bessel beam remain separated indefinitely. Furthermore, when the optical diffraction is taken into account, the effective diffusion coefficient becomes a complex number $\left(D+i V_{g} / 2 / q_{1}\right)$, with the group-velocity determining the ratio between the real (pure-diffusion) and the imaginary (diffraction) parts. Such complex diffusion can possibly be useful for all-optical image processing, such as image enhancement, denoising and edge-detection [39].

Ramsey narrowing occurs when the pump's crosssection is finite, and atoms that re-enter the beam from outside, less affected by power-broadening, contribute to the spectrum. Our model gives simple and analytic results for the Ramsey-narrowed spectrum by solving a diffusion equation with spatially dependent decay coefficients and sources. It is somewhat surprising that our steady-state approach is able to capture this effect, for any pumping and transit rates, so that one is not required to average over atomic trajectories. Utilizing our model, the spectrum for any detailed geometry can readily be obtained.

The theory presented here may contribute to the analysis of decoherence in collective light memories, for which it was shown that the decay rate is proportional to the single-atom diffusion rate [40]. The model can potentially be extended to include the main ground-state decoherence mechanisms in vapor: non-coherence-preserving collisions, namely spin-exchange collisions, and wall col- 
lisions. Such extensions may aid in developing vapor EIT schemes with narrower lines.

\section{Acknowledgments}

This work was partially supported by DDRND and the fund for encouragement of research in the Technion.

[1] E. Fermi, Rev. Mod. Phys. 4, 87 (1932).

[2] R. H. Dicke, Phys. Rev. 89, 472 (1953).

[3] L. Galatry, Phys. Rev. 122, 1218 (1961).

[4] M. Nelkin and A. Ghatak, Phys. Rev. 135, A4 (1964).

[5] D. Budker, L. Hollberg, D. F. Kimball, J. Kitching, S. Pustelny, and V. V. Yashchuk, Phys. Rev. A 71, 012903 (2005).

[6] G. Dutier, A. Yarovitski, S. Saltiel, A. Papoyan, D. Sarkisyan, D. Bloch, and M. Ducloy, Europhys. Lett. 63, 35 (2003).

[7] G. C. Corey and F. R. McCourt, J. Chem. Phys. 81, 2318 (1984).

[8] A. D. May, Phys. Rev. A 59, 3495 (1999).

[9] E. Arimondo, "Coherent Population Trapping in Laser Spectroscopy", Progress in Optics (Elsevier, Amsterdam, 1996), vol. 35.

[10] J. J. Longdell, E. Fraval, M. J. Sellars, and N. B. Manson, Phys. Rev. Lett. 95, 063601 (2005).

[11] K. J. Boller, A. Imamoglu, and S. E. Harris, Phys. Rev. Lett. 66, 2593 (1991).

[12] L. Hau, S. E. Harris, Z. Dutton, and C. H. Behroozi, Nature 397, 594 (1999).

[13] M. D. Lukin, Rev. Mod. Phys. 75, 457 (2003).

[14] S. E. Harris and L. V. Hau, Phys. Rev. Lett. 82, 4611 (1999).

[15] N. Cyr, M. Tetu, and M. Breton, IEEE Transactions on Instrumentation and Measurement 42, 640 (1993).

[16] S. Knappe, V. Shah, P. D. D. Schwindt, L. Hollberg, J. Kitching, L.-A. Liew, and J. Moreland, Applied Physics Letters 85, 1460 (2004).

[17] P. D. D. Schwindt, S. Knappe, V. Shah, L. Hollberg, and J. Kitching, Applied Physics Letters 85, 6409 (2004).

[18] O. Firstenberg, M. Shuker, A. Ben-Kish, D. R. Fredkin, N. Davidson, and A. Ron, Phys. Rev. A 76, 013818 (2007).

[19] A. Nagel, C. Affolderbach, S. Knappe, and R. Wynands, Phys. Rev. A 61, 012504 (1999).

[20] C. Bolkart, D. Rostohar, and M. Weitz, Phys. Rev. A 71, 043816 (2005).

[21] M. Shuker, O. Firstenberg, R. Pugatch, A. Ben-Kish,
A. Ron, and N. Davidson, Phys. Rev. A 76, 023813 (2007).

[22] E. Pfleghaar, J. Wurster, S. I. Kanorsky1, and A. Weis, Opt. Comm. 99, 303 (1993).

[23] I. Novikova, Y. Xiao, D. F. Phillips, and R. L. Walsworth, Journal of Modern Optics 52, 2381 (2005).

[24] Y. Xiao, I. Novikova, D. F. Phillips, and R. L. Walsworth, Phys. Rev. Lett. 96, 043601 (2006).

[25] N. F. Ramsey, Phys. Rev. 78, 695 (1950).

[26] E. Buhr and J. Mlynek, Phys. Rev. Lett. 57, 1300 (1986).

[27] A. S. Zibrov, I. Novikova, and A. B. Matsko, Opt. Lett. 26, 1311 (2001).

[28] A. S. Zibrov and A. B. Matsko, Phys. Rev. A 65, 013814 (2001).

[29] T. Zanon, S. Guerandel, E. de Clercq, D. Holleville, N. Dimarcq, and A. Clairon, Phys. Rev. Lett. 94, 193002 (2005).

[30] R. Pugatch, M. Shuker, O. Firstenberg, A. Ron, and N. Davidson, Phys. Rev. Lett. 98, 203601 (2007).

[31] M. Shuker, O. Firstenberg, R. Pugatch, A. Ron, and N. Davidson (2007), quant-ph/0707.3707.

[32] S. Chandrasekhar, Rev. Mod. Phys. 15, 1 (1943).

[33] M. D. Lukin, S. F. Yelin, and M. Fleischhauer, Phys. Rev. Lett. 84, 4232 (2000).

[34] S. Chapman and T. G. Cowling, The Mathematical Theory of Non-Uniform Gases (Cambridge University Press, 1960).

[35] F. Zimmer, A. Andre, M. Lukin, and M. Fleischhauer, Opt. Comm. 264, 441 (2006).

[36] A. E. Siegman, Lasers (University Science Books, California, 1986).

[37] W. Gawlik, Phys. Rev. A 34, 3760 (1986).

[38] J. Durnin, J. J. Miceli, and J. H. Eberly, Phys. Rev. Lett. 58, 1499 (1987).

[39] G. Gilboa, N. Sochen, and Y. Y. Zeevi, IEEE Trans. Pat. Anal. Machine Intel. 26, 1020 (2004).

[40] C. Mewes and M. Fleischhauer, Phys. Rev. A 72, 022327 (2005). 